Literaturnachweis

CARLE, W.: Die Lagerungsstörungen in den Kieselgurgruben von Ohe (Lüneburger Heide). - Abh. Nat.-Ver. Bremen, 31, 1939.

DEWERS, F.: Diluvium (in „Das Känozoikum in Niedersachsen“). - Geol. u. Lagerst. Niedersachsens 3. Teil, Oldenburg i. O. 1941.

DÜCKER, A.: Die Windkanter des norddeutschen Diluviums in ihren Beziehungen zu periglazialen Erscheinungen und zum Decksand. - Jahrb. preuß. geol. Landesanstalt, 54, 1933.

KAY, G. F. und PEARCE, J. N.: The origin of Gumbotil. - Journ. of Geology, 28. 1920.

RICHTER, K.: Stratigr. u. Entwicklungsgesch. mittelpomm. Tertiärhöhen. - Abh. u. Ber. Pomm. Natf. Ges. 1927.

SIMON, W. G.: Geschiebezählungen und Eisrandlagen in Südost-Holstein. - Mitt. d. geogr. Ges. Lübeck, 39, 1937.

TROLL, C.: Strukturböden, Solifluktion u. Frostklimate der Erde. - Geol. Rundschau, 34, 1944.

WOLDSTEDT, P.: Ưber die Ausdehnung der letzten Vereisung in Norddeutschland. Ber. Reichsamtes f. Bodenf. Wien, 1942.

\title{
Die geologische und archäologische Stellung des altpaläolithischen Fundplaţes Markkleeberg bei Leipzig
}

Von Rudolf G r a h m a n n, Bielefeld. Mit 3 Abb.

Die im Jahre 1895 durch den Leipziger Geologen FRANZ ETZOLD (1918) entdeckte und seither fast ununterbrochen ausgebeutete altpaläolitische Fundstätte Markkleeberg bei Leipzig ist die am längsten bekannte und noch immer die reichste ihrer Art in Deutschland. Sie fand ihre erste Bearbeitung durch K. H. JACOB (FRIESEN), der im Jahre 1910 die von ETZOLD nicht bekannt gegebene Fundstelle nochmals entdeckte, und durch C. GÄBERT (JACOB (FRIESEN) und GÄBERT, 1914). JACOB (FRIESEN) unterschied unter dem Einfluß französischer Prähistoriker nach Art und Technik drei archäologische Stufen, deren älteste er für oberes Acheuléen oder unteres Mousterien hielt, deren jüngste er dagegen als Hochmousterien mit Anklängen an Aurignacien ansah. Der Geologe C. GÄBERT kam zu dem Schluß, daß die Fundschicht in die zweite Hälfte oder eher an das Ende des vorletzten Interglazials zu stellen sei. Da es sich erwies, daß das französische Mousterien allgemein dem Ende des letzten Interglazials und dem Beginn des Würmglazials angehört, ergab sich für Markkleeberg ein Widerspruch zwischen archäologischer und geologischer Altersbestimmung; K. BRAUNE (1929 und 1933) sprach von einer Problematik der Fundstelle.

Es erschien daher nötig, Fundstelle und Funde einer erneuten Bearbeitung zu unterwerfen. Geologisch kam dieser zugute, daß mittlerweile die Gliederung der pleistozänen Ablagerungen Sachsens bis ins Einzelne durchgeführt war (GRAHMANN 1925 und 1934); und archäologisch einerseits der Ausbau des Gliederungssystems hauptsächlich durch H. BREUIL, andererseits das Anwachsen des Markkleeberger Fundstoffes. Dieser war allerdings in einer Menge öffentlicher und privater Sammlungen zerstreut. Er wurde in den Jahren 1936 bis 1940 aufgespürt und karteimäßig festgehalten. Es wurden dabei mehr als 3000 Stücke erfaßt, davon die weitaus meisten von Markkleeberg selbst, ein kleiner Teil von benachbarten gleichartigen Fundstellen. Auf Grund dieses für eine altpaläolitische Fundstätte in Deutschland beispiellos reichen Fundstoffes 
konnte dessen archäologische Stellung viel genauer und sicherer erfaßt werden als vorher.

Eine im Dezember 1944 abgeschlossene Monographie behandelt erschöpfend sowohl die geologische wie die archäologische Stellung des Fundplatzes, unterstützt durch 15 Textabbildungen und 131 Tafeln, auf denen mehr als 500 Stücke in je mehreren Ansichten abgebildet werden. Das erste Manuskript ging bei dem Verlag während der Besetzung am Ende des Krieges verloren. Ein neues Manuskript wurde im Sommer 1947 vom Urbanverlag, Freiburg i. Br., angenommen. Es ist zu hoffen, daß die Arbeit im Jahre 1951 erscheinen wird. Die folgenden Ausführungen können sich nur auf das wichtigste beschränken, und zwar auch nur auf die Fundstätte Markkleeberg allein. Für die ebenfalls wichtigen gleichartigen Fundplätze bei Leipzig ebenso wie für die als Grundlage des gesamten deutschen Altpaläolithikums dienenden Abbildungen der größtenteils hervorragend schönen Werkstücke muß auf die Monographie selbst verwiesen werden.

In der Umgebung von Leipzig sind mit Sicherheit d re i Eiszeiten festzustellen, doch hat nur in der Mindel- und in der Rißeiszeit das nordische Inlandeis das Gebiet überschritten. Die Schichtfolgen dieser beiden Glaziale entsprechen einander. Sie beginnen jeweils mit Flußschottern, die 6 bis $15 \mathrm{~m}$ mächtig sind. Auf ihnen liegt, in der mindelglazialen Schichtfolge fast ausnahmslos, in der rißglazialen hier und da, ein Bänderton als Absatz des Staubeckens, das sich jeweils vor dem Eisrande auf dem gegenhängigen Talboden bildete und vom Eise bei seinem Vordringen nach Süden talaufwärts geschoben wurde. Das Stauwasser entlastete das Inlandeis dabei so, daß es häufig fast schwerelos über den fetten Ton hinwegglitt, ohne ihn zu stauchen. Auf ihm lagerte sich dann die Grundmoräne ab. Sie wird hier und da von Schmelzwasserkiesen und Sanden bedeckt.

Die Flußschotter der beiden älteren Eiszeiten unterscheiden sich durch ihren Geschiebegehalt. Die Mindelschotter führen ausschließlich einheimische Gerölle aus dem südlichen Einzugsgebiet der Flüsse, die Rißschotter dazu auch nordische Geschiebe, die aus zerstörten Mindelmoränen stammen. Da die Mindelvereisung die größte Ausdehnung hatte und sehr mächtige Ablagerungen hinterließ, so sind nordische Geschiebe, insbesondere Feuersteine, in den rißglazialen Schottern meist recht reichlich vertreten.

In der Würmeiszeit wurden ebenfalls Schotter abgelagert, außerdem auch der Löß angeweht. Die Südgrenze des echten mächtigen Lößes liegt $20 \mathrm{~km}$ südlich von Markkleeberg, doch ist ihr ein etwa $30 \mathrm{~km}$ breiter Saum von stratigraphisch gleich zu bewertendem höchstens $1 \mathrm{~m}$ mächtigen Sandlöß oder Flugsand überall nordwärts vorgelagert (R. GRAHMANN, 1932).

Die Markkleeberger Fundstücke finden sich in ri B glazialen Schottern der Pleiße, eines rechten Nebenflußes der Weißen Elster. Das hier durch einige Kiesgruben aufgeschlossene Profil zeigt zu oberst eine $0,4 \mathrm{~m}$ starke Decke von Sandlöß als alleinigen Vertreter des Würmglazials. An seiner Basis liegt immer eine dichte Steinsohle aus vorwiegend nordischen Geschieben, die zum großen Teile Windschliff aufweisen. Es handelt sich um den Rest der im letzten Interglazial abgetragenen einstigen Grundmoräne des Rißglazials, die südlich von Leipzig sonst bis zu $2 \mathrm{~m}$ stark ist und auch in den Markkleeberger sowie benachbarten Gruben fetzenweise unter dem Löß noch erhalten ist. Ein Bänderton ist in Markkleeberg nicht zu beobachten. Vielmehr liegen unter der Steinsohle waagrecht geschichtete Flußsande, etwa $1 \mathrm{~m}$ stark, die nach unten in sandigen Flußkies übergehen. Diese Sande und Kiese sind eine völlig einheitliche Bildung, 
die der rißglazialen Aufschotterung im Pleißetale entspricht und hier, nahe dem Uferrande der Schotterterrasse, insgesamt $4 \mathrm{~m}$ mächtig ist. Die oberen Sande sind meistens kryoturbat verwulstet, und zwar bereits im Rißglazial, mitunter auch etwas glaziär gestaucht. Selten ist auch eine würmglaziale Verbrodelung $\mathrm{zu}$ bemerken, an der also, vor Beginn der Lößanwehung, auch die Steinsohle teilgenommen hat. Sand und Kies sind im letzten Interglazial verwittert und verlehmt, daher braun und ziemlich standfest. An ihrer Basis liegen große Geschiebe aus der Mindelmoräne. Das Liegende bilden miozäne Süßwassersande.

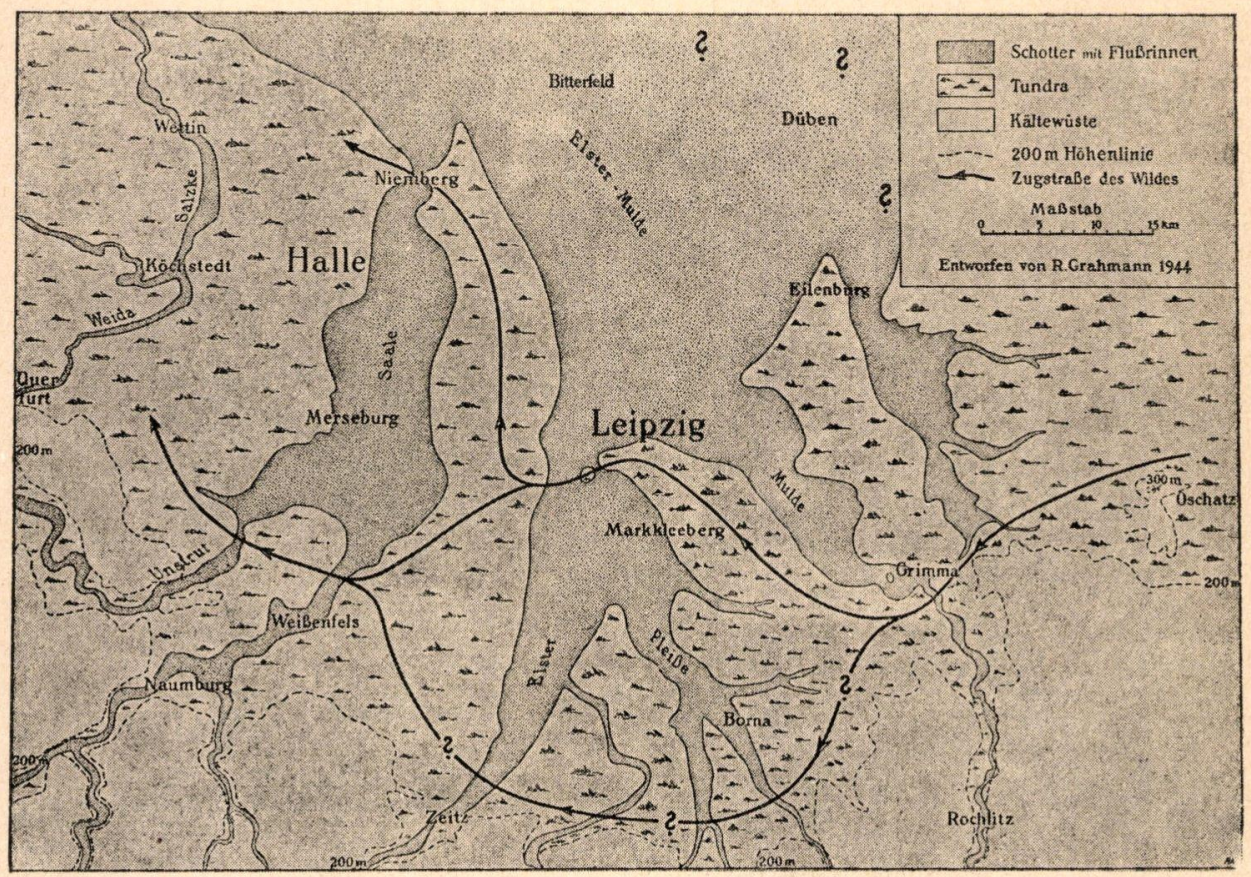

Abb. 1: Rißglaziale Schotterflächen und Landschaftsformen bei Leipzig.

Die Stellung der Markkleeberger Pleißeschotter als $\mathrm{r}$ i B glazial ist unzweifelhaft. Sie ergibt sich auch aus dem Terrassenlängsprofil, aus dem Zusammennange der Terrasse mit den gleichalten Terrassen der Nachbarflüsse Mulde und Elster, die bei Leipzig sich zu einer sehr weiten Schotterfläche vereinigen, aus der Fauna, und, wenigstens klimatisch, auch aus der Flora. Die weiten, im Hochglazial wahrscheinlich völlig sterilen Schotterflächen schrieben dem schweifenden Wilde und damit seinem Jäger die Bahn vor, wie Abb. 1 zeigt. Diese läßt auch erkennen, daß im Westen von Leipzig eine Enge im Schotterzuge der Elster offenbar dem Wilde als bevorzugter Úbergang diente, woraus sich die Anwesenheit des Menschen und die Häufung paläolithischer Funde am Ostrande der Schotterebene südlich von Leipzig erklärt.

Die Schotter haben an vielen Stellen, so auch in Markkleeberg, Elephas primigenius, E. trogontherii, Rhinoceros tichorhinus, Rangifer arcticus geliefert, also eine durchaus glaziale Fauna, die durch den im Riß aussterbenden Elephas trogontherii auch zeitlich bestimmt ist. Eine bei Böhlen, $8 \mathrm{~km}$ südwestlich von Markkleeberg, dem Pleißeschotter eingeschaltete Tonbank führte die Reste einer echten Tundrensumpffiora, von Holzgewächsen nur dünne Zweige kleinwüch- 
siger Weiden und an Pollen nur äußerst selten Kiefer, Birke und Weide, die entweder kleinen Tundrenformen angehören oder auch von Ferne eingeweht sein können (R. GRAHMANN 1924). Jedenfalls erweist sich durch diese Flora eine waldfreie, tundraartige Landschaft schon bald nach Beginn der Aufschotterung.

Die Werkstücke finden sich im Markkleeberger Pleißeschotter regellos eingestreut und werden beim Abbau des Kieses gefunden. Wohl ist von manchen Sammlern die Beobachtung gemacht worden, daß diese oder jene Schicht zeitweise ergiebiger war. Es konnte aber trotz allen Bemühungen niemals eine archäologisch faßbare Abfolge festgestellt werden. Vielmehr finden sich Stücke der verschiedensten Formen, Arten und Erhaltungszustände sowohl im unteren wie im oberen Teile des Kieses, seltener in den hangenden Sanden. Das ist nach der Natur der Lagerstätte nicht zu verwundern. Die rißglazialen Pleißeschotter umfassen, geologisch gesprochen, eine kurze Zeitspanne, vielleicht ein Jahrzehntausend. Und der Werkbestand ist die Hinterlassenschaft schweifender Horden, die in zeitlichen Abständen von Jahrhunderten, vielleicht Jahrtausenden, am Ufer der Pleiße rasteten, jagten und ihre Werkzeuge schlugen. Sie entstammten möglicherweise verschiedenen Kulturkreisen, und was uns von ihnen in Markkleeberg erhalten blieb, ist sonach ein zeitlicher Querschnitt durch die Kulturen des frühen Rißglazials, also archäologisch anders zu bewerten, als der Werkbestand eines nur kurze Zeit besiedelten Wohnplatzes.

Von den rund 3000 Fundstücken bestehen nur vier aus alttertiärem Süßwasserquarzit, alle anderen aus baltischem Feuerstein verschiedener Art. Die weitaus meisten dieser Werkstücke zeigen denselben $\mathrm{Erh}$ alt ung z $\mathrm{ust}$ and wie die jetzigen als Gerölle in dem Schotter vorkommenden Feuersteine, d. $h$. sie sind im allgemeinen gebräunt. Dies gilt für ungerollte und scharfe Stücke ebenso, wie für gescheuerte oder abgerollte. Alle diese Werkstücke sind zweifellos gleich alt wie der Schotter. Sie sind offenbar kurz nach ihrer Verfertigung in diesen geraten und bei dem Aufschotterungsvorgange bald eingebettet worden. Die Rollung ist absolut kein Gradmesser für das Alter, wie man das leider immer noch hier und da lesen kann, sondern sie zeigt zunächst lediglich an, daß ein Stück längere Zeit im Flusse verfrachtet worden ist. Das kann ein Stück, das südlich, also flußoberhalb von Markkleeberg, während der Aufschotterungszeit geschlagen wurde, recht gut sein, es ist dann doch ebenso alt wie ein scharfes, in Markkleeberg geschlagenes.

Es gibt unter den Fundstücken jedoch auch solche von einem durchaus abweichenden Erhaltungszustande. Sie sind meistens stark abgerollt und haben eine dicke weiße, porzellanartige Rinde. Diese Werkstücke sind zweifellos älter als die oben genannten. Sie wurden wahrscheinlich in der dem Rißglazial vorausgehenden Warmzeit am Flusse geschlagen, wo man den Feuerstein als Rohstoff auflas, gerieten bald nach der Verfertigung in den Fluß, wurden von diesem verfrachtet und dabei abgerollt, bis sie, am Gleithange einer Flußschlinge aufs Trockene geworfen, hier unter dem Einflusse von Luft und Niederschlägen tief weißlich patinierten. Erst viel später, als der Fluß sein Bett durch Aufschotterung wieder erhöhte, gelangte̊n sie abermals in dieses, wurden mitunter von neuem bestoßen und gerollt und erlitten später im Grundwasser manchmal auch eine mehr oder minder tiefe Braunfärbung ihrer Porzellanpatina.

Die zuletzt genannten, höchstwahrscheinlich prärißischen Werkstücke machen im Markkleeberger Fundstoffe nur $2 \%$ aus. Gerollte rißglaziale Stücke sind mit $31 \%$ vertreten, ungerollte mit $67 \%$. Es wurden also auch flußoberhalb von Markkleeberg Werkstücke geschlagen, in Markkleeberg selbst aber weitaus am meisten. 
Der Franzose COUTIER hat an Feuersteinen Versuche über die Sch lag te $\mathrm{chnik}$ im Paläolithikum angestellt, die erlauben, einen Fundstoff zu gliedern. Darüber wird in der oben erwähnten Monographie ausführlich berichtet. Hier kann nur soviel gesagt werden, daß das Abschlagen der Werkstücke vom rohen Feuerstein oder von einem vorbereiteten Kern entweder Stein gegen Stein erfolgte (harter Schlag) oder mit Holz, Knochen, vielleicht auch weicherem Gestein (weicher Schlag). Der harte Schlag ist in grober Weise durch Anschlagen gegen einen $A m b o ß$ während des Abbeville und Clacton, in feiner Weise mit einem Klopfstein im Acheul angewendet worden. Er erzeugt stets einen Schlagkegel mit anschließender mehr oder minder kräftiger Schlagzwiebel. Bei weichem Schlag entsteht kein Schlagkegel, auch der Schlagbuckel ist meistens nicht sehr ausgeprägt, er zeigt nahe der Schlagstelle fast immer eine kleine Aussplitterung. Diese Technik führt zu längeren oder dünneren Abschlägen als der harte Schlag. Sie ist bezeichnend für das Levallois, in dem harter Schlag nur selten angewendet worden ist. Ähnliche Ergebnisse werden bei Anwendung eines Holzoder Knochenmeißels erzielt, einer Technik, die wohl besonders im Jungpaläolithikum üblich war, aber wahrscheinlich mitunter auch schon in Markkleeberg angewendet worden ist. Das Levallois unterscheidet sich von den anderen Kulturen und Techniken dadurch, daß die Schlagfläche an dem meistens schildkrötenförmigen Kern (tortoise core) durch einige Retuschen rechtwinklig zugerichtet wird, die dann auf der Basis des Abschlages sogenannte Fazetten bilden. Doch ist dies nicht bei allen Stücken des Levallois der Fall. Die Technik des Levallois ist später auch in anderen Kulturkreisen angewendet worden, beispielsweise häufig im Moustier.

Unter den Markkleeberger Stücken des vorletzten Interglazials sind $78 \%$ mit hartem Schlage und nur $22 \%$ mit weichem Schlage bei einflächiger Basis hergestellt. Dagegen zeigt der rißglaziale Fundstoff nur $34 \%$ mit hartem Schlag, aber $42 \%$ mit weichem Schlag auf einflächiger Basis und $24 \%$ mit weichem Schlag auf fazettierter Basis. Daraus spricht ein entschiedener Fortschritt in der Herstellung langer und dünner Abschläge, also in der Richtung auf Klingenerzeugung.

Eine solche anteilsmäßige Betrachtung der Technik scheint für alle altpaläolithischen Fundplätze wichtig. Die Ergebnisse lassen sich anschaulich in einem gleichseitigen Dreieck darstellen, das grundsätzlich dem in der Petrographie üblichen Osann'schen Dreieck entspricht. In einem Dreieck, wie die Abb. 2 es zeigt, würde der Punkt unten rechts $100 \%$ Stücke mit hartem Schlag, also ein reines $\mathrm{Clac}$ ton bedeuten, wogegen die Spitze des Dreieckes einem natürlich nur theoretischen Levallois mit $100 \%$ fazettierten Schlagflächen ent-

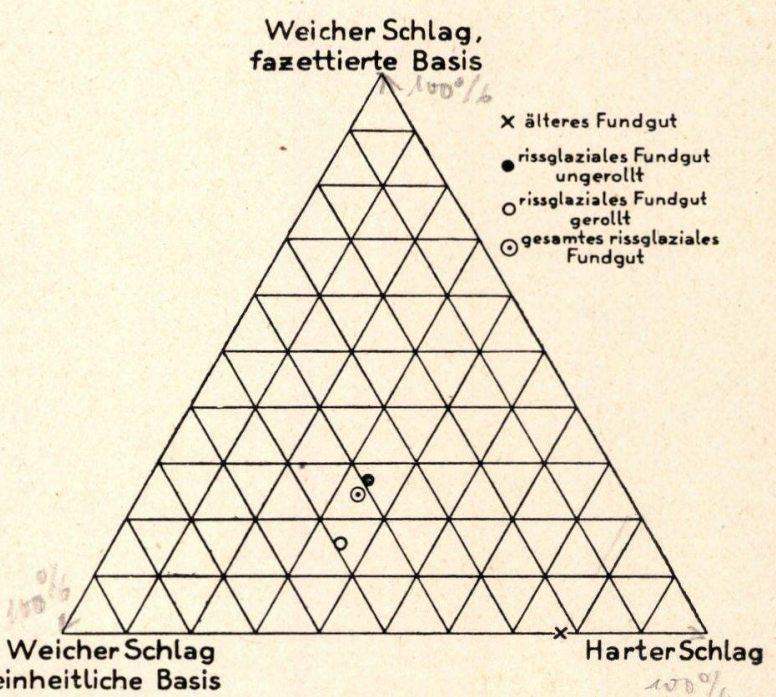

Abb. 2: Anteil der Schlagweisen im Fundgute von Markkleeberg. 
sprechen würde. Die Punkte für die älteren und die rißglazialen Markkleeberger Stücke zeigen deren Stellung gegenüber den genannten reinen Kulturen.

Über die in Markkleeberg übliche $\mathrm{Nacharbeit} \mathrm{kann} \mathrm{hier} \mathrm{nur} \mathrm{soviel}$ gesagt werden, daß die weitaus meisten Stücke eine künstliche Schartung der Arbeitskanten, oder Schneiden aufweisen. Mitunter ist sie wechselseitig als Sägung, mitunter so dicht, daß sie als einfache Retusche in einem Arbeitsgang bezeichnet werden kann. Vollretusche in mehreren Arbeitsstufen ist ziemlich selten, doch sind dabei sehr vollkommene Stücke, wie schon Abbildungen in der Monographie von JACOB und GÄBERT (1914) beweisen. Auch Wegretuschierung der Schlagzwiebel, Ausschlagen von Buchten, Zuarbeitungen zu Spitzen, Zinken und Nasen sind vertreten.

Wie aus den verschiedenen Ausführungen bereits hervorgeht, überwiegen in dem Markkleeberger Werkbestande die aus Abschlägen verschiedener Technik hergestellten Werkstücke durchaus. Ihnen gegenüber spielen aus dem Kern geschlagene Stücke eine völlig untergeordnete Rolle. Es kann hier nicht eine ins Einzelne gehende Darstellung und Beurteilung der Werkzeugtypen und -arten gegeben werden. Dafür wird auf die im Druck befindliche Monographie verwiesen. Zum Verständnis der archäologischen Schlußfolgerungen sei über den Werkbestand nur das folgende gesagt:

Es sind in Markkleeberg zu unterscheiden:

1. Kerne; als solche werden alle Feuersteinstücke angesehen, von denen Abschläge gewonnen worden sind. Meistens werden die Kernsteine durch vorbereitende Schläge hergerichtet. Bei der Technik des Levallois ist dies immer der Fall. In Markkleeberg sind festzustellen Diskuskerne, Schildkerne, Walzenoder Prismenkerne, Spindelkerne. Der Anteil der Kerne am Gesamtfundstoff ist gering. Er macht nur knapp 3\% der rißglazialen Fundstücke aus, was möglicherweise darauf zurückzuführen ist, daß die meisten Sammler die Kernsteine in dem an groben Feuersteinen reichen Schottern nicht beachtet haben.

2. Grobger äte; sie sind fast ausschließlich mit hartem Schlag gewonnen, meistens wohl am Amboß in Clactontechnik und weisen dann die entsprechenden Merkmale auf, große einflächige Basis, kräftigen aus einem Schlagkegel entspringenden Schlagbuckel, offenen Schlagwinkel von meistens $110^{\circ}$ bis $120^{\circ}$, mitunter bis $135^{\circ}$. Manche der häufig handgroßen, ja bis zu mehr als $3 \mathrm{~kg}$ schweren, groben Abschläge lassen eine gewollte Formgebung nicht erkennen, doch beweist die künstliche Schartung der Schneiden ihre Benutzung als Werkzeug. Die meisten Grobgeräte jedoch haben beabsichtigte Formen, die immer wiederkehren, aber wegen ihrer dicken klobigen Gestalt unter den üblichen Werkzeugformen nicht unterzubringen sind. Die Grobgeräte machen $8 \%$ des gesamten rißglazialen Werkstückbestandes von Markkleeberg aus; im älteren Fundgut sind sie mit $15 \%$ vertreten.

3. Klingen und Klingenabschläge spielen in Markkleeberg die Hauptrolle. Im rißglazialen Werkbestande sind sie mit fast 59\% führend; dasselbe gilt für den älteren Fundstoff, in dem sie mit fast $55 \%$ vertreten sind. Die Klingen sind in verschiedenen Arbeitsweisen hergestellt worden. Einerseits gibt es Übergänge zu den Grobgeräten, andererseits durch Anwendung des Meißelschlages Anklänge an das Jungpaläolithikum. Recht häufig ist die Technik des Levallois. Die längsten Klingen erreichen mehr als $14 \mathrm{~cm}$ Länge, die meisten sind 5 bis $6 \mathrm{~cm}$ lang. Das Verhältnis von Breite zu Länge kann kleiner sein als 1:4; im Durchschnitt liegt es bei $1: 2$. Die meisten Klingen zeigen künstliche Schartung, auch Sägung. Einschneidig und zweischneidig retuschierte 
Klingen sind recht selten. Außer ihnen sind zu erwähnen Klingen mit seitlicher Bucht, Klingen mit Querschneide, mit Bohrerspitze und Schrägendklingen, die anscheinend als Vorläufer der jungpaläolithischen Stichel anzusehen sind.

4. B r e it a b s chläg e sind mindestens so breit wie lang, meistens breiter. Sie treten in runden, ovalen und viereckigen Formen auf und bilden rund $51 / 2 \%$ des rißglazialen Bestandes. Levalloistechnik ist bei ihnen häufig zu erkennen.

5. Spitzen; in dieser Gruppe sind, wie bei den Klingen, alle Arbeitsweisen vertreten. Dickspitzen schließen an ähnliche Formen der Grobgeräte an. Außerdem werden unterschieden Klingenspitzen, Fünfeckspitzen, beide häufig nach der Arbeitsweise des Levallois hergestellt, ferner viel selteñer Spitzen mit Seitenbucht, ein- und zweischneidig retuschierte Spitzen und, besonders bemerkenswert, sehr selten Stielspitzen. Die Spitzen bilden insgesamt fast $12 \%$ der rißglazialen Werkstücke, fast $15 \%$ der älteren.

6. Sch a ber sind nur solche Stücke genannt worden, die bei entsprechender Form die durchgehende Retuschierung, seltener geschlossene Schartung mindestens einer Schneide aufweisen. Nach dieser strengen Begriffsbestimmung ist die Zahl der Schaber in Markkleeberg nicht sehr groß. Sie machen nur etwa 5\% unter den rißglazialen Werkstücken aus, sind aber zum Teil von schönster Arbeit. Einige weisen Rasten für den Daumen oder für eine Schäftung auf.

7. $\mathrm{Kratzer}$ mit meist steil retuschierter Arbeitskante sind in verschiedenen Formen als Kiel-, Stirn-, Hohl- und Buchtkratzer vertreten. Wie manche Schaber zeigen sie keinen Schlagteil, sei es, daß dieser abgetrennt wurde, oder daß es sich um natürliche Feuersteinscherben handelt. Die Kratzer sind allgemein größer als die des Jungpaläolithikums. Unter den rißglazialen Werkstücken von Markkleeberg machen sie fast $8 \%$ aus.

8. B ohrer, Nase n, Z a cke n, Gerätearten, die im Jungpaläolithikum eine große Rolle spielen, sind auch im Altpaläolithikum bereits vertreten, allerdings meistens in größeren Formen. In Markkleeberg sind sie, wie die Kratzer, meistens ohne Schlagteil und bilden etwa $2 \%$ des gesamten rißglazialen Werkzeugbestandes.

9. Doppels eiter; einen zerbrochenen, ursprünglich wahrscheinlich mandelförmigen Faustkeil von Markkleeberg hat bereits JACOB (FRIESEN) (1914) abgebildet. Einen weiteren gab später F. WIEGERS bekannt. Beide Stücke sind ziemlich die besten ihrer Art von Markkleeberg. Was sonst an Doppelseitern dort gefunden worden ist, zeigt meistens eine ungeschickte Bearbeitung und beweist, daß die Verfertiger kaum geübt waren, solche Stücke zu schlagen. Diese sind vorwiegend aus Abschlägen hergestellt worden. Unter den rißglazialen Werkzeugen finden sich nur 13, im älteren Fundgute nur ein Doppelseiter.

Der gesamte Werkbestand von Markkleeberg, insbesondere der geringe Ánteil von Doppelseitern, beweist einwandfrei, daß es sich sowohl bei dem älteren als auch bei dem rißglazialen Fundgute um A b s hla g kulturen handelt. Es werden unter diesen, meist recht willkürlich, reine Abschlagkulturen, Handspitzenkulturen, Breit- und Schmalklingenkulturen, Schaberkulturen und ähnJiche unterschieden. Eine solche Zuordnung kann aber nicht allein aus den Bearbeitungsweisen und Arten der Geräte hergeleitet werden, sondern muß auch die Mengenanteile beachten. Dieser bei Beurteilung jungpaläolithischer oder mesolithischer Fundplätze meistens beachtete Grundsatz hat bei uns für das Altpaläolithikum noch keine Anwendung gefunden. Die Fülle des Markkleeberger Fundgutes aber erlaubte, wie wir sahen, eine solche Statistik. Sie zeigte, daß man in erster Linie von einer $\mathrm{K}$ ling e $\mathrm{n}$ kultur sprechen kann, ob schmal oder breit, 
hängt natürlich von der Begriffsbestimmung ab. Die in Druck befindliche Monographie bringt Längen-Breiten-Streubilder.

Außer den Klingen sind in Markkleeberg jedoch auch andere Werkzeuggruppen vertreten, die bei der Beurteilung mit berücksichtigt werden sollten. Läßt man die urtümlichen Grobgeräte beiseite, so lassen sich die übrigen Formen zwanglos in drei Hauptgruppen einteilen, nämlich einerseits die Klingen und Breitabschläge, dann die Spitzen, und schließlich die Schaber, Kratzer, Bohrer und Doppelseiter gemeinsam. Die Geräte dieser letzten Gruppe sind immer retuschiert, die der anderen meistens nicht. Die Doppelseiter können hier mit einbezogen werden, weil sie überwiegend aus Abschlägen hergestellt sind und ihre Zahl kaum ins Gewicht fällt. Wo sie jedoch zahlreich auftreten und aus dem Ganzen hergestellt wurden, bleiben sie bei einer solchen Betrachtungsweise besser außer Ansatz.

Die auf diese drei Gruppen beschränkte Aufteilung des rißglazialen Werkbestandes ergibt für die Klingen $70 \%$, für die Spitzen $13 \%$ und für die letzte

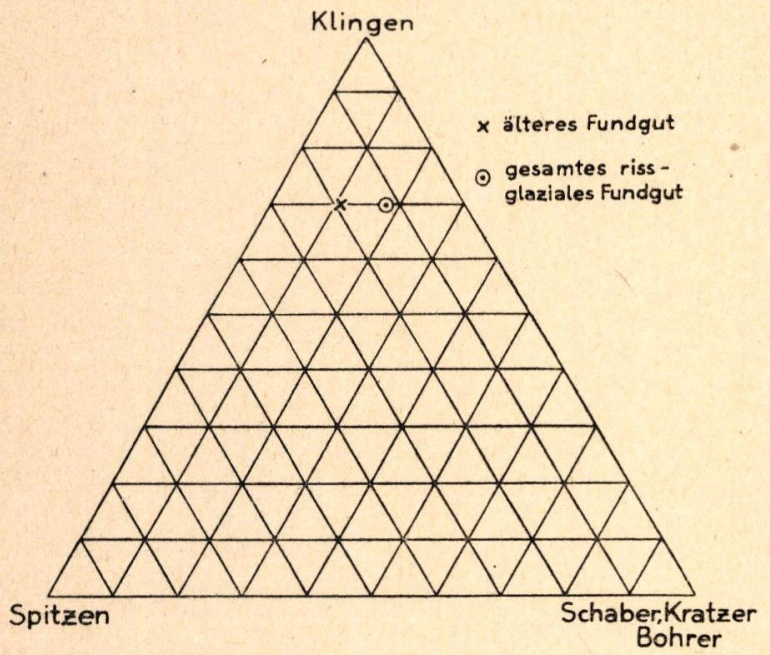

Abb. 3: Anteil der wichtigsten Gerätegruppen im Fundgute von Markkleeberg. Gruppe $17 \%$. Unter den nur 36 in Betracht kommenden älteren Stücken bilden die Klingen ebenfalls $70 \%$. Spitzen sind mit $19 \%$, Schaber mit $11 \%$ vertreten. Schlüsse lassen sich aus diesem geringfügigen Unterschied nicht ziehen, zumal feinretuschierte Stücke, wie etwa Kratzer und Zacken bei der meistens starken Abrollung der älteren Stücke nicht erkannt werden und daher unterbewertiet bleiben. Die Stellung der Geräteanteile im gleichseitigen Dreieck ist in Abb. $3 \mathrm{zu}$ erkennen.

Bei der Beantwortung der Frage, welcher archäologischen Stufe die Funde von Markkleeberg zuzuordnen seien, muß an die eingangs schon ausgesprochene Tatsache erinnert werden, daß wir es hier, wie bei allen Schotterfundplätzen, nicht mit der Hinterlassenschaft eines einzigen Wohnplatzes zu tun haben, sondern mit dem etwa ein Jahrzehntausend umfassenden Querschnitt der dinglichen Kulturen jener Zeit. Es kann also sehr wohl möglich sein, in dem Fundgute ein Gemenge mehrerer Kulturen $\mathrm{zu}$ sehen, die, geologisch gesprochen, gleichzeitig in Mitteldeutschland vertreten waren. Die verschiedenen Arbeitsweisen, die großen Unterschiede etwa zwischen den Grobgeräten und den sehr fein gearbeiteten Spitzen und sonstigen retuschierten Stücken machen diese Annahme sogar wahrscheinlich.

Es war schon vorn darauf hingedeutet worden, daß die Markkleeberger Grobgeräte überwiegend die Schlagmerkmale des Clacton aufweisen. Aber auch ihre Formen entsprechen zum Teil denen von Clacton on Sea und anderen reinen Clactonfundplätzen. Außer den Grobgeräten können auch manche Dick- 
spitzen und eine Reihe plumper Klingen zum Clacton gerechnet werden, sodaß dessen Anteil am rißglazialen Fundgute mit etwa $10 \%$ zu bewerten ist.

Die Stücke mit fazettierten Schlagflächen müssen alle dem L e v a 11 o is zugerechnet werden, aber nicht nur sie, sondern fast alle mit weichem Schlage gewonnenen Abschläge, zumal sie meistens die für das Levallois übliche, beim Herrichten der Schild- und Walzenkerne entstehende Oberflächenbehandlung zeigen. Auch an den klassischen Fundplätzen des Levallois in Nordfrankreich tragen nicht alle Abschläge retuschierte Basisflächen. Es errechnet sich so für unseren rißglazialen Fundstoff ein Mindestanteil des Levallois von $60 \%$.

Der Rest von rund $30 \%$ ist archäologisch am schwersten zu fassen. Technisch zeigt er entweder die Merkmale des Acheuls, nämlich den Abschlag mit einem harten Stein (was bis zur Jungsteinzeit geübt wurde); oder es fehlt ein Schlagteil überhaupt. Dies ist aber bei den gleichen Werkzeugformen des französischen Acheul häufig auch der Fall. Unter dieser Gruppe finden sich die meisten gut retuschierten Stücke des gesamten Fundgutes. Wenn man berücksichtigt, daß im französischen Altlevallois retuschierte Stücke kaum vertreten sind und erst, zweifellos unter dem Einfluß des Acheuls, im jüngeren Levallois erscheinen, so hat man doch den Eindruck, daß auch in Markkleeberg sowohl die Schlagtechnik wie die Vorliebe für Retuschierung, schließlich das Auftreten wenn auch meistens ungeschickt geschlagener Doppelseiter auf Einfluß des A che uls zurückzuführen sind.

Der älte re Markkleeberger Fundstoff ist wegen seines meist schlechten Erhaltungszustandes auf diese Weise schwer zu beurteilen. Der Anteil des Acheuleinflusses mag ebenfalls $30 \%$ betragen. Clacton und primitives Levallois mögen etwa zu gleichen Teilen vertreten sein. Stücke mit fazettierten Basen sind zwar aus Markkleeberg nicht bekannt, jedoch von einem in der Nähe gelegenen völlig gleichartigen Fundplatze.

Als Ganzes schält sich nunmehr für die Fundstätte Markkleeberg heraus, daß hier aus dem Rißglazial wie aus der vorhergehenden Warmzeit Gemenge des Clacton, des Levallois und des Acheul vorliegen. $\mathrm{Zu}$ Beginn der Rißeiszeit ging in Mitteldeutschland die Entwicklung des Altpaläolithikums dahin, anstelle der im Clacton üblichen, auf dem Amboß geschlagenen klobigen Grobgeräte bessere und feinere mit Knochen gehauene Werkzeuge zu schaffen, was mehr und mehr eine sorgfältige Herrichtung des Kernes und der Schlagfläche erforderte. So entwickelte sich aus der Abschlagkultur des Clacton die erste Klingenkultur des älteren Levallois. Zu diesem reinen Entwicklungsgange gesellte sich von außen her eine Beimengung oder ein Einfluß des Acheuls, in dem eine geschickte Schlagweise mit dem Klopfstein und meistens sorgfältige Retuschierung üblich waren. Man kann also sagen, der rißglaziale Fundst off von Markkleeberg ist ein klingenreiches unteres Levallois mit Resten von Clacton und einem Einschlag von mittlerem Acheul.

Der ältere Fundstoff zeigt soviele Ähnlichkeit mit dem rißglazialen, daß er als dessen unmittelbarer Vorläufer angesehen werden muß. Die Schlagweise des Levallois wurde erst von wenigen angewandt, der Anteil des Acheul beruht vermutlich zum Teil auf Beimengung. Trotz dem einschneidenden Klimawechsel bei Beginn der Rißeiszeit lassen sich in Markkleeberg keine kulturellen Verschiebungen feststellen, die auf $\mathrm{Ab}$ - oder Zuwanderungen hinweisen. Vielmehr scheint es, daß auch in der Mindel-Riß-Warmzeit Mitteldeutschland im Wesentlichen von Horden durchstreift wurde, die der großen Gruppe der Abschlagkulturen angehörten, wogegen die Vertreter der afrikanisch-westeuropäischen 
Faustkeilkulturen nur vereinzelt und selten nach dem Osten vorstießen. Sie waren warmen Klimaten angepaßt und konnten offenbar kalte kontinentale Winter ebensowenig ertragen, wie das Flußppferd, dessen interglaziale Ostgrenze im allgemeinen mit der der geschlossenen Faustkeilkulturen zusammenfällt. Eine Ausnahme bilden eigentlich nur die ziemlich reichlichen zusammen mit echten Levalloisabschlägen auftretenden Zweiseiter der südlichen Umgebung von Hannover; doch ist deren Alter leider geologisch nicht sicher festzustellen; typologisch scheinen sie zum Teil jünger als Riß zu sein.

$\mathrm{Da} B$ umgekehrt besonders in Kaltzeiten Vertreter der östlichen Abschlagkulturen nach Westen vorstießen, sodaß in Frankreich und England Verzahnungen der beiden großen Kulturkreise erfolgten, ist nach Lage der Dinge als durchaus selbstverständlich anzusehen. Dabei kam es in England und in Belgien während des beginnenden Rißglazials zu Verschmelzungen oder Vermengungen, die als Early Mousterian und als Mesvinien gewisse Ähnlichkeiten mit Markkleeberg aufweisen. Eine Wiederholung desselben Vorganges zu Beginn des Riß-Würmglazials hatte dann das echte Mousterien zur Folge. Aus der Ähnlichkeit dieses Ablaufes folgt die Ähnlichkeit der Ergebnisse und damit der Erklärung, warum früher die Markkleeberger Funde, ebenso wie die südenglischen der Rißzeit, als Moustierstufe angesprochen worden sind.

Vieles konnte im Rahmen eines Vortrages nur gestreift werden. Für weitere Ausführungen und besonders für die das deutsche Altpaläolithikum erschöpfend erfassenden Abbildungen muß auf die demnächst erscheinende Monographie hingewiesen werden.

\section{Erwähnte Schriften}

BRAUNE, K.: Das Problem der Paläolithen von Markkleeberg bei Leipzig. - Sber. naturforsch. Ges. Leipzig, 53/55, 1929. - Zur Problematik der paläolithischen Fundstätte Markkleeberg. - Mannus 25, 1933.

ETZOLD, F.: Beiträge zur Kenntnis der Steinzeit bei Leipzig und bei Merseburg. Sber. naturforsch. Ges. Leipzig 43/44, 1918.

GRAHMANN, R.: Über pflanzenführende Diluvialtone in Nondwestsachsen. - Z. dtsch. geol. Ges. 76. 1924. - Diluvium und Pliozän in Nordwestsachsen. - Abh. Math. Phys. Kl. d. Sächs. Akad. d. Wiss. 39, Nr. 4, 1925. - Der Löß in Europa. Mitt. Ges. Erdkde. Leipzig, 1932. — *) L'âge géologique de l'industrie paléolithique de Markkleeberg. - L'Anthropologie 45, Paris 1935. - Grundriß der Quartärgeologie Sachsens, in FRENZEL - RADIG - RECHE: Grundriß der Vorgeschichte Sachsens. - Leipzig 1934. - ${ }^{*}$ ) Die altpaläolithische Fundstätte Markkleeberg und weitere gleichartige Fundplätze bei Leipzig. Geologisch-archäologische Monographie mit 15 Abb. im Text und 131 Tafeln. Im Druck.

JACOB, K. H. ${ }^{*}$ ) und GABERT, C.: Die altsteinzeitliche Fundstelle Markkleeberg bei Leipzig. - Veröff. Städt. Mus. f. Völkerkde. zu Leipzig, Heft 5, Leipzig 1914.

*) Die mit einem *) gekennzeichneten Arbeiten bringen Abbildungen Markkleeberger Werkstücke. 